\title{
Craniomandibular Disorder
}

National Cancer Institute

\section{Source}

National Cancer Institute. Craniomandibular Disorder. NCI Thesaurus. Code C84654.

A term referring to disorders affecting the muscles of the head and neck, particularly the masticatory muscles. 